\title{
A RADICAL FOR NEAR-RINGS
}

\author{
W. E. DESKINS
}

1. Introduction. In a recent paper [1], D. W. Blackett extended a number of results from the theory of semisimple rings to semisimple near-rings. Whereas a semisimple ring is ordinarily defined as being a ring from which the (or a) radical has been "deleted," in [1] a semisimple near-ring was defined without a radical-like ideal being introduced first. It is the purpose of this note to demonstrate the existence of an ideal $R$ in a near-ring $N$ such that the difference near-ring $N-R$ is semisimple (in the sense of Blackett). The ideal $R$ will be the zero ideal if and only if $N$ is semisimple. Furthermore, if $N$ is a ring, then $R$ will be the usual nilpotent radical. Thus it seems appropriate to call $R$ the radical of $N$.

Here, as in [1], attention is restricted to those near-rings which satisfy the descending chain condition for right modules and the requirement that the zero element of the near-ring annihilates the near-ring from the left. Terminology not defined here may be found in $[1]$.

2. Definition of $R$. Let $M$ be a minimal right module of $N$, and let $r(M)$ be the set of all elements of $N$ which annihilate $M$ from the right. Clearly $r(M)$ is an ideal (two-sided) of $N$. Now let $\mathcal{X}$ be the set of all minimal right modules of $N$ and define $S$ to be intersection of all the $r(M), M \in \mathcal{X}$. Therefore $S$ is an ideal of $N$.

Theorem 1. $N$ is semisimple if and only if $S$ is the zero ideal.

Let $S$ be the zero ideal. Now $N$ was defined to be semisimple if it contained no nilpotent right modules [1], so let $Q$ be a nilpotent right module of $N$. It may be assumed without loss of generality that $Q$ is a minimal right module of $N$. Since $Q^{2}=0$ and since $S=0$, there exists another minimal right module of $N$, say $P$, such that $P Q \neq 0$. Hence there exists an element $p \in P$ such that $p Q=P$. But since $p Q^{2}=0=P Q$, we see that $N$ is semisimple.

Now suppose $N$ is semisimple. Since $S$ is an ideal of $N$ it is a right module and so contains $P$, a minimal right module of $N$. But $S \subseteq r(P)$ so that $P^{2}=0$, and it follows that $S$ must be the zero ideal.

\section{Theorem 2. The near-ring $N_{1}=N-S$ is semisimple.}

Let $N_{1}$ contain the nilpotent minimal right module $Q_{1}$, and let $Q$

Presented to the Society, May 1, 1954; received by the editors February 3, 1954. 
be the pre-image of $Q_{1}$ in $N$. Then $Q$ is a right module of $N$, and $Q^{2} \subseteq S$. If $Q \nsubseteq S$, then there is a minimal right module $M_{1}$ of $N$ such that $M_{1} Q \neq 0$. Hence $M_{1}$ contains an element $m$ such that $m Q=M_{1}$. But $M_{1} Q=(m Q) Q \subseteq m S=0$, and so $Q \subseteq S$. Therefore $Q_{1}=0$ and $N_{1}$ is semisimple.

It will be illustrated later by a simple example that $S$ may be a larger ideal than desired. Consequently the following definition of $R$ must be given.

First, let $P$ be the set of all ideals $P$ of $N$ which contain no nonzero idempotents but which contain at least one nonzero element which annihilates all idempotents of $N$ from the left. Then define $T$ to be the sum of all $P \in P$ (that is, the set of all sums of a finite number of elements chosen arbitrarily from the ideals $P$ ). If $P$ is void, define $T$ to be the zero ideal.

Now form $S$, as previously defined, in the near-ring $N_{1}=N-T$, and let $\bar{N}=N_{1}-S$. Then we define $R$, the radical of $N$, to be the kernel of the homomorphism $\sigma: N \rightarrow \bar{N}$.

TheOREM 3. The near-ring $N-R$ is semisimple, and $N$ is a semisimple near-ring if and only if $R$ is the zero ideal.

This is an immediate consequence of the preceding results and the structure theory for semisimple near-rings [1].

3. The ring case. To justify calling $R$ the radical of $N$, the following is necessary.

Theorem 4. If $N$ is a ring, then $R$ coincides with the usual radical.

The chain condition for right modules becomes the chain condition for right ideals when $N$ is a ring. Thus

$$
\begin{aligned}
N & =e_{1} N+\cdots+e_{n} N+r(e) \\
& =N e_{1}+\cdots+N e_{n}+l(e),
\end{aligned}
$$

where $e_{i} N$ and $N e_{i}$ are indecomposable right and left ideals, the $e_{i}$ are primitive idempotents, $e$ is the sum of the $e_{i}$, and $r(e)$ and $l(e)$ are the sets of all elements of $N$ which annihilate $e$ from the right and left respectively. Therefore $T$ is nilpotent and $N_{1}=N-T$ contains a right identity element. Clearly the ideal $S$ of $N_{1}$ is also nilpotent, so that if $K$ is the usual radical of $N, R \subseteq K$. Since $N-R$ contains no nilpotent right ideals, $R \supseteq K$. Hence $R=K$.

It should be pointed out that this definition of $R$ is essentially an adaptation of the arithmetic characterization of the radical of a ring with identity element given by N. Jacobson [3]. 
REMARK 1. The necessity of introducing the ideal $T$ is demonstrated by the following example. Let $N$ be the ring having basis elements $a$ and $b$ over the rational field, where multiplication is defined as follows: $a^{2}=a, a b=b, b a=b^{2}=0$. The only minimal right ideal of $N$ is the principal ideal $(b)$, and therefore $S=r(b)=N$. However, $R=(b)$.

REMARK 2. It was recently pointed out to the author that the ideal $S$ was very similar to the definition of a radical for rings given by O. Goldman [2]. However this similarity is somewhat superficial due to a perhaps unfortunate choice of terminology. A right module, as defined in [1], becomes nothing more than a right ideal if $N$ is a ring, whereas a right module in ring theory is considerably more general than a right ideal. Hence our Theorem 4 is not a consequence of Goldman's work.

\section{BIBLIOGRAPHY}

1. D. W. Blackett, Simple and semisimple near-rings, Proc. Amer. Math. Soc. vol. 4 (1953) pp. 772-785.

2. O. Goldman, $A$ characterization of semisimple rings with the descending chain condition, Bull. Amer. Math. Soc. vol. 52 (1946) pp. 1021-1027.

3. N. Jacobson, The theory of rings, Mathematical Surveys, no. 2, New York, American Mathematical Society, 1943, p. 66.

The Ohio State University 